\title{
Attentats de 2015 en France: sécurité globalisée et repli diplomatique
}

\author{
JEAN JACQUES KOURLIANDSKY*
}

Pour citer cet article : Kourliandsky, J. J. (2016). Attentats de 2015 en France : sécurité globalisée et repli diplomatique. Desafios, 28(II), 413-424.

La France, l'un des cinq membres permanents du Conseil de sécurité a, comme tous les Etats de ce cercle, cultivé la dimension extérieure de sa politique. La France a été victime en 2015 d'une série d'attentats. Ces attentats ont une origine extérieure avouée. Ils ont en effet été revendiqués par l'organisation Etat islamique (EI). La base territoriale de cette organisation terroriste est située au Proche-Orient, en Irak et en Syrie. Mais ces crimes collectifs ont été commis pratiquement tous par des nationaux français et belges, le 7 janvier comme le 13 novembre 2015.

La réaction des autorités françaises a pris une double dimension intérieure et extérieure, en cohérence avec ce double défi terroriste. Elle a combiné une intervention militaire en Syrie et l'adoption de mesures judiciaires, policières et militaires sur le territoire national. Bien qu'inscrites dans un cadre évolutif engagé déjà depuis plusieurs années, perceptibles dans les derniers Livres blancs de la défense ${ }^{1}$, les initiatives réactives adoptées en 2015 et en 2016, ont pour la première fois posé les fondements d'un système sécuritaire global et intégré.

\footnotetext{
* Institut des relations internationales et stratégiques. Paris, France. Courriel : jkourliandsk@gsan.org

1 Livre blanc, défense et sécurité nationale, Paris, La documentation française, 2013.
} 
De façon explicite cette approche efface les frontières qui traditionnellement séparent sécurité intérieure et sécurité extérieure, politique intérieure et politique extérieure. La menace, tous azimuts, brouille les logiciels politiques. ${ }^{2}$ Au nom de la nécessaire protection de la vie elle oriente les actions publiques vers une réduction des libertés inhérentes aux systèmes démocratiques et vers le recours à la force et non à la diplomatie pour résoudre les crises internationales.

Les manuels de relations internationales admettaient bien depuis longtemps la perméabilité croissante des souverainetés étatiques. Ces traités avaient également progressivement ouvert l'éventail des acteurs de la vie internationale. Pour autant, l'existence d'un intérieur et d'un extérieur, paraissait garder toute sa pertinence. Classique du genre l'Introduction à l'histoire des relations internationales, de Pierre Renouvin et Jean-Baptiste Duroselle, définit « l'étude des relations internationales comme l'analyse des relations entre communautés politiques organisées dans le cadre d'un territoire, c'est-à-dire entre les Etats »(Pierre Renouvin, 1991).

Publié en 2010, un ouvrage plus récent valide le point de vue. « La politique étrangère, est l'instrument par lequel l'Etat tente de façonner son environnement politique international» (...) «L'effort d'une société nationale de contrôler son environnement externe », " pour maximiser ses intérêts et minimiser les risques » (Tshiyembe, 2010).

Pourtant de plus en plus souvent aujourd'hui, sécurité intérieure et questions de défense se croisent, se mêlent, jusqu'à devenir un objet sécuritaire unique. Cette extension de l'insécurité et des réponses qui lui sont apportées efface de façon universelle la différence entre politique extérieure et politique intérieure. Le phénomène n'est pas propre à la France. Il concerne aussi ses voisins européens et méditerranéens comme les Etats américains, du nord et du sud.

\footnotetext{
2 Questions internationales, «Les nouveaux espaces du jihadisme », Paris, $\mathrm{n}^{\circ} 75$, septembre
} 2015 . 
La résurgence de doctrines de sécurité globale, réactualisant les pensées de sécurité nationale, héritées d'une autre époque, accompagne ces évolutions. On assiste à une combinaison insolite entre ce qui a pu être décrit par une équipe d'historiens comme un phénomène de « repli » national, sur une exaltation de la force légale, unifiant ses instruments intérieurs et extérieurs (Bancel, Blanchard \& Boubeker, 2015). Ce repli suppose-t-il une répétition d'événements liés à la sécurité nationale dans les années 1970-1980?

Un défi est en tous les cas posé aux Etats démocratiques soumis aux aléas de flux transfrontaliers, et victimes en même temps d'agressions terroristes d'origine tant extérieure qu'intérieure, un défi qui a effacé les genres diplomatiques, militaires et policiers traditionnellement séparés. La relève quasi simultanée des ministres français des Affaires étrangères et de la Justice, Laurent Fabius et Christiane Taubira, en janvier et février 2016, a matérialisé la prééminence prise par les ministères et les corps de violence légale, Intérieur et Défense.

Faut-il voir là la réponse d'un Etat agressé par un terrorisme sans frontières? S'agit-il d'une résurgence des doctrines de sécurité nationale ? S'agit-il d'une opportunité sécuritaire saisie par un Etat aux compétences érodées par la mondialisation des économies, de la finance et de l'information?

L'examen de la conjoncture particulière à la France, permet en tous les cas de poser une problématique. Une problématique, sans doute localisée et spécifique mais qui peut ouvrir la perspective d'analyses comparatives plus générales.

\section{Disparition des frontieres fonctionnelles entre securite interieure et exterieure}

Le premier constat qui interpelle l'observateur des derniers événements ayant affecté la France en 2015, les attentats et les réactions des pouvoirs publics à ces attentats, est celui d'un mélange des genres assumé. La réponse aux crimes a produit des décisions sécuritaires combinant aspects internes comme extérieurs. 
Le pays a été déclaré le 16 novembre 2015 par le président français, François Hollande, en état de guerre. L'armée, instrument de préservation de la souveraineté extérieure, a donc été mobilisée à l'extérieur, mais aussi sur le territoire national. Plusieurs milliers de militaires participent en France à des opérations de sécurité intérieure. Ils seraient, selon des chiffres officiels, environ 10 000. Ils patrouillent dans les aéroports, les gares. Ils sécurisent les lieux de cultes, essentiellement juifs et musulmans, et leurs extensions culturelles. Ils participent à la protection des édifices publics de souveraineté : présidence, ministères, assemblées législatives.

Ces décisions ne relèvent pourtant pas de la conjoncture terroriste de ces derniers mois. Elles constituent l'aboutissement d'une évolution relativement ancienne. Les forces armées sont mobilisables en France pour des actions sécuritaires depuis 1978. Ce recours aux soldats porte un nom, le plan Vigipirate. ${ }^{3}$ Cette mobilisation sur le sol national est doublée d'interventions extérieures en Afrique et en Orient, dans les foyers extérieurs du terrorisme : en République centrafricaine, en Irak, en Libye, au Mali, en Syrie.

Le dispositif avait été initié dans un quasi anonymat administratif. Il avait été légitimé politiquement dans les Livres blancs de la défense nationale ${ }^{4}$. Plusieurs lois antiterroristes avaient par ailleurs été adoptées après chaque épisode de violence aveugle. «Sécurité et liberté », intitulé d'un premier projet de loi adopté en 1980, visait après un attentat palestinien contre une synagogue, à réduire au nom de la sécurité collective les libertés publiques. Les attentats de 2015 ont ouvert un espace nouveau légitimant de façon globale les mesures d'exception démocratique déjà adoptées au nom de l'efficacité antiterroriste.

L'état d'urgence, dispositif légalisé par une loi du 3 avril 1955, pendant la guerre d'Algérie, a été mis en œuvre au lendemain des attentats du

\footnotetext{
3 Lire Le Monde, Paris, 8 septembre 2015, p.11.

4 Les derniers Livres blancs en français s'intitulent « Défense et sécurité nationale ». Ils font référence au rôle de la police nationale et au plan «Vigipirate » pour assurer la défense du pays.
} 
13 novembre 2015, le 14, prolongé de trois mois, législativement, le 20 du même mois. D’une durée de trois mois ce dispositif a été prolongé d'une autre période de trois mois, à dater du 26 février 2016. Le recours à l'état d'urgence a fait l'objet d'une volonté de constitutionnalisation de la part du gouvernement. Il a été accompagné d'une proposition parallèle de destitution de la nationalité française pour les binationaux auteurs de crimes terroristes.

La mise en examen sans délit constaté de personnes considérées comme suspectes en raison de déplacements dans les pays ou territoires foyers de groupes terroristes a été légalisée. Le philosophe Régis Debray a commenté cette évolution, pour lui paradoxale, dans l'hebdomadaire brésilien Epoca: «Aujourd’hui les jeunes européens ne partent plus pour la Bolivie, mais pour la Syrie et l'Irak. La seule vraie contestation à la domination capitaliste des Etats-Unis est celle de Ben Laden et de l'Etat islamique $\gg{ }^{5}$

Les mesures proposées aujourd'hui ont ouvert un chantier juridique qui bouscule le droit. Il s'agit en effet pour le juge de sanctionner un individu considéré comme potentiellement dangereux avant qu'il ait commis un éventuel délit. ${ }^{6} \mathrm{La}$ délation est par ailleurs encouragée. Les surveillants de prison sont invités à noter les comportements de détenus entrant dans la grille des comportements considérés comme potentiellement dangereux.

A l'extérieur, en Afrique, au Proche-Orient, au nom d'une légitime défense reposant sur une interprétation extensive du droit international, diverses opérations ont été engagées par la France depuis $2007^{7}$. Un droit particulier, le droit d'ingérence humanitaire, droit dérogatoire, a été créé à cet effet. En 2011, pour intervenir en Libye afin de

\footnotetext{
5 In Epoca, San Pablo, 17 août 2015.

6 Voir Marc Trevidic, Terroristes, Paris, Lattés, 2013.

7 Opérations extérieures en cours au 1er février 2016 : Barkhane dans le Sahel africain, environ 3500 soldats ; Daman au Liban, 500 militaires ; Chammal en Irak-Syrie, 2700 hommes; Sangaris en Centrafrique 900 personnels. Par ailleurs sont positionnés 1000 militaires en Côte d'Ivoire ; 1000 à Djibouti ; 350 au Gabon ; 350 au Sénégal.
} 
protéger les populations menacées dans l'est du pays, à Benghazi, ce droit à protéger signalé dans la résolution 1711 du Conseil de sécurité a par exemple été instrumentalisé et interprété de la façon la plus large afin de procéder à un changement de régime.

Le président des Etats-Unis, George Bush, a proposé une autre dérogation au droit international, validée par les nécessités de sécuriser son pays. Le 20 septembre 2002, il a théorisé une conception de la sécurité intérieure légitimant les actions extérieures préventives au nom d'une suspicion agressive. Construite après les attentats ayant visé New York et Washington le 11 septembre 2011, cette philosophie sécuritaire défend la nécessité d'intervenir préventivement hors des Etats-Unis pour répondre au défi posé sur le territoire national par le crime terroriste. ${ }^{8}$

Le débat qui a été ouvert après ces attentats a marginalisé de fait la diplomatie. La concertation internationale anti-terroriste a pris le pas sur la concertation et la fabrication de compromis négociés. Le ministère français des Affaires étrangères a concentré son travail en 2015 sur deux dossiers éloignés de ces questions: la tenue à Paris de la conférence sur le réchauffement climatique (la COP21) et la diplomatie économique et commerciale.

Policiers et militaires, leurs ministres respectifs, pendant la même période, ont multiplié les déplacements internationaux liés au combat contre le terrorisme. Un débat a été ouvert dans les corps dont ils ont la responsabilité entre ceux qui souhaitent maitriser les dérogations au droit et ceux qui au contraire veulent élargir les évolutions sécuritaires encadrant l'exercice des libertés démocratiques. Un certain nombre de militaires ont ainsi exprimé leur attente d'une norme leur permettant d'exercer des pouvoirs jusqu’ici réservés à la police. ${ }^{9}$ Les

\footnotetext{
8 «L'action préventive prime la dissuasion », Washington, usinfo.state.gov, 24 septembre 2002.

9 Voir dans la « Revue Défense nationale», n786, janvier 2016, Hervé de Bonnaventure, «Les armées au cœur de la protection du territoire national », pp. 22-28
} 
syndicats de police de leur côté ont exprimé le vœu de pouvoir utiliser leurs armes avec davantage de flexibilité. ${ }^{10}$

\section{Construction d'un nouvel ennemi global}

Cette fusion du domaine sécuritaire est-elle la réponse d'Etats débordés par la globalisation, ayant recours à la force comme ultime argument permettant de légitimer leur pertinence? Ou s'agit-il d'autre chose, un « autre chose » qui lié à la pression du terrorisme renvoie les Etats, la France en l'occurrence ici à son passé de guerres coloniales, aux dérogations au droit qui à l'époque avaient été tolérées par les nécessités de la lutte contre les nationalistes algériens, alors qualifiés de terroristes?

La France, comme bien d'autres Etats est entrée dans une dynamique internationale différente dans les dernières années du XXème siècle. Une dynamique résumée par Bertrand Badie de la façon suivante. « La disparition de la guerre froide, les effets perturbateurs et novateurs de la décolonisation et de la mondialisation, ont fait leur œuvre » (Badie, 2013). Les flux de toute sorte ont débordé les Etats. Qu'il s'agisse d'informations, de devises, de marchandises, ou du crime organisé, quelles que soient ses motivations.

La contestation des souverainetés, par porosités croissantes, a certes encouragé des formes d'organisation collective. Les unions douanières, les ententes intergouvernementales, le droit des relations internationales ont connu une expansion parallèle à celle des flux les plus diversifiés. Mais les délégations de souveraineté, loin de permettre de relever les défis posés aux Etats, ont accentué une évolution de moins en moins maitrisée.

La crise économique qui affecte la France et la plupart de ses voisins est restée sans réponse. Elle bouscule une société composite. La richesse croise en effet les origines nationales les plus diverses. Des

\footnotetext{
10 Voir « la liste musclée des envies des policiers », in Le Monde, 6-7 décembre 2015, p. 13.
} 
centaines de milliers de migrants ont été importés pendant «les trente glorieuses ", d'Afrique du nord, d'Afrique noire, de Turquie et des Antilles. Ils ont fait souche et sont restés en dépit de la dégradation du marché du travail.

Faute de pouvoir créer du lien collectif par la croissance et la création d'emplois, les discours officiels et partisans ont dérivé en direction de l'identité. A défaut de sorties positives de la crise, les autorités ont offert à certains de leurs gouvernés mécontents des boucs émissaires, les derniers arrivés sur le sol national, étrangers par définition au dénominateur commun collectif. Le recours à la force contre un ennemi tout à la fois extérieur et intérieur dans un tel contexte a pu apparaitre comme l'ultime recours, permettant aux Etats de légitimer ce qu'ils sont, les dépositaires autorisés de la violence légale. Ce serait selon les critiques des théories réalistes le révélateur d'un comportement paradoxal, exprimant par le recours à la force, «l'impuissance de la puissance $»{ }^{11}$

Armées, police, sont utilisées conjointement pour des opérations de sécurité intérieure, et pour des opérations de sécurité extérieure, visant selon ses initiateurs à écraser les foyers du crime (Mathias, 2015), situés de façon indifférenciée dans les banlieues françaises, en Syrie et en Afrique sahélienne. Un ennemi tout à la fois extérieur et intérieur a été défini et identifié ${ }^{12}$ de façon répétitive. L'ennemi désigné et diabolisé, comme «terroriste » l'est aussi et de plus en plus comme « étranger». Cette évolution suppose une orientation porteuse de ce qu'une équipe d'historiens français a qualifié comme un « grand repli» (Bancel, Blanchard \& Boubeker, 2015).

\section{Retour de la securite nationale et/ou diplomatie limitee?}

La sécurité intégrale, articulant sécurité extérieure et sécurité intérieure, police et armée, avait été théorisée dans les années 1960. La

\footnotetext{
11 «Bertrand Badie, L'impuissance de la puissance »

12 Voir Mathieu Rigouste (2011).
} 
politique de sécurité nationale visait à combattre de façon coordonnée l'ennemi intérieur défini au service, réel ou potentiel, d'un adversaire extérieur déstabilisant. Les décisions prises par les autorités françaises s'inscrivent en apparence dans le droit fil de cette théorie politique et sécuritaire. La doctrine sécuritaire théorisée et mise en pratique dans les années 1960-1970 répondait à une urgence politique, celle de préserver un statu quo interne et extérieur.

Mais l'ennemi d'hier, justifiant le recours à une violence d'Etat exceptionnelle, relevait d'un contexte qui n'existe plus, celui de la guerre froide et de la décolonisation. ${ }^{13}$ L'ennemi extérieur, l'Union soviétique, a disparu pour des raisons sans rapport avec les contre-feux policiers et militaires inventés en France et en Amérique latine. L'Algérie a conquis son indépendance nationale. Les forces nationalistes de gauche participent à la vie politique et souvent gouvernementale de nombreux pays latino-américains ayant connu la dictature.

L'application de théories sécuritaires a généré des dérives démocratiques autoritaires, voire des régimes de dictature dans le passé. Le pouvoir a été en Amérique latine accaparé dans les années 1960-1980 par des régimes autoritaires et dictatoriaux. Les libertés démocratiques ont été encadrées pendant les guerres coloniales en France. Qualifiés de terroristes, les défenseurs de la démocratie, quelles que soient leurs orientations idéologiques, ont été en Amérique latine arrêtés, souvent tués, après condamnation et parfois exécutés hors de toute décision judiciaire. ${ }^{14}$ En France crime et torture ont été tolérés par les gouvernements au nom de la raison d'Etat. Cette perte de repères éthiques et politiques a conduit la IVème République à se saborder le 13 mai 1958.

Aujourd'hui la réalité politique est aussi celle de discours et de pratiques qui encadrent de plus en plus les libertés démocratiques. Ce qui rappelle et renvoie aux doctrines de sécurité nationale du siècle dernier. Au nom de la sécurité citoyenne, menacée par un ennemi

\footnotetext{
13 Voir note 9.

14 Voir Hélio Bicudo (1984).
} 
extérieur, ayant des ramifications sur le sol national, les gouvernements et les parlements adoptent des lois réduisant le champ des libertés. Ils restreignent le recours à la diplomatie, et privilégient le recours aux opérations militaires. Le constat que l'on peut faire est celui d'un encadrement croissant ${ }^{15}$ des libertés et de pratiques dérogatoires au droit international.

Il convient pourtant de relativiser la comparaison entre les évènements actuels et ceux d'hier. Comparaison est d'autant moins raison que les circonstances historiques d'aujourd'hui, celles de la mondialisation, ne sont pas et ne sont plus celles de la guerre froide et des guerres coloniales. Les réalités internationales actuelles sont celles d'un défi collectif posé aux Etats par la mondialisation. Ce défi touche les Etats traditionnellement considérés comme " centraux », par les théoriciens de la « dépendance ». ${ }^{16}$ Dans un tel contexte le domaine de la «périphérie » affecte toute sorte d'Etats quelle que soit leur idéologie affichée.

Ce qui conduit à relativiser les critiques croisées sur le mauvais usage des principes universels de la démocratie. Critiques adressées aux gouvernements néo-libéraux, jugés liberticides par les Etats «bolivariens ». Critiques ciblant les gouvernements «bolivariens » et assimilés adressées par les pays dirigés par des « libéraux ». Le constat ici avancé est celui d'une convergence des réponses. La conjoncture particulière de chacun définit la modalité de la réaction et de l'initiative. Les uns et les autres ont inventé des stratégies permettant de contenir la « périphérisation » de l'Etat. Côté cour, celui des démocraties libérales comme côté jardin, celui des démocraties «bolivariennes».

Les attentats de 2001 aux Etats-Unis ont été à l'origine de pratiques démocratiques d'exception : Patriot act, interventions militaires en Afghanistan, en Irak, en Syrie, accompagnées de crimes d'Etat, commis dans l'anonymat d'avions sans pilote. Dario Battistella a rappelé, peu après «l'évènement apocalyptique » du 11 septembre 2001, les

\footnotetext{
15 Voir les livres de Laurent Mucchielli et Roland Gori (2013).

16 Celso Furtado et Fernando Henrique Cardoso.
} 
ambiguïtés de la «notion de sécurité $»{ }^{17}$ la France a suivi le même cheminement comme cela a été signalé. Les Etats «bolivariens » et assimilés ont pour leur part prétendu récupérer leur souveraineté contestée en repoussant de façon autoritaire les ingérences « centrales »: des lois restrictives ont été appliquées pour restreindre le droit de propriété des groupes économiques étrangers, des entreprises médiatiques, et de leurs relais locaux.

Le fil directeur de ces décisions ambiguës ou en apparence incompatible, est peut-être à explorer dans d'autres directions. Le diplomate et sociologue latino américaniste français Alain Rouquié a défini les régimes argentin et vénézuélien des Kirchner et Chavez-Maduro, comme des " démocraties hégémoniques ${ }^{18}$ ». Le concept peut être élargi. Il est susceptible de prendre une dimension universelle et tout aussi bien s'appliquer à la France, aux Etats-Unis, à la Colombie et au Mexique, si l'on fait abstraction des différences idéologiques évidentes de leurs gouvernements respectifs.

Tous ces Etats et leurs gouvernements, en effet, se considèrent démocratiques. Pourtant tous ont instrumentalisé une menace sécuritaire particulière, -terrorisme, narcotrafic, puissances économiques privées-, pour réduire l'espace de dialogue, intérieur comme extérieur. On assiste à une fusion croissante des fonctions de sécurité extérieure et intérieure, des armées et de la police, un recours à la violence légale, qui réduisent les compétences traditionnelles du juge et du diplomate. La «démocratie hégémonique» pratiquée par les uns et par les autres réduit l'espace des contre-pouvoirs, de négociations, et de compromis, l'esprit des lois des Etats modernes. Ce qui ne suppose pas nécessairement une dérive vers la reconstitution d'Etats reposant sur le principe de la sécurité nationale du siècle passé. Mais ce glissement institutionnel est révélateur d'une incapacité globale à trouver par d'autres voies que celles de la violence, fût-elle légale, une issue aux crises d'aujourd'hui.

17 Dario Battistella, «Théorie des relations internationales », Paris, Presses de Sciences Po, 2003.

18 In Le Monde, 20-21 décembre 2016, p. 19, « Le pouvoir use, surtout si on en abuse ». 


\section{Références}

Badie, B. (2013). L'impuissance de la puissance. Paris: Biblis-CNRS.

Bancel, N., Blanchard, P. \& Boubeker, A. (2015). Le grand repli. Paris: L'Harmattan.

Bicudo, H. (1984). Segurança nacional ou Submissão. Rio de Janeiro: Paz e Terra. Mathias, G. (2015). La guerre française contre le terrorisme islamiste. Des confins du Sahara à nos banlieues. Paris: Giovanangeli.

Mucchielli, L. \& Gori, R. (2013). La fabrique des imposteurs. Babel.

Pierre Renouvin, J.-B. D. (1991). Introduction à l'bistoire des relations internationales. Paris : Armand Collin.

Rigouste, M. (2011). L'ennemi intérieur. Paris: La Découverte.

Tshiyembe, M. (2010). Géopolitique mondiale. Paris: L'Harmattan. 